\title{
MicroRNA-20a contributes to cisplatin-resistance and migration of OVCAR3 ovarian cancer cell line
}

\author{
YANKUN LIU ${ }^{1}$, SUGUI HAN ${ }^{2}$, YUHUI LI ${ }^{3}$, YAN LIU ${ }^{4}$, DI ZHANG $^{1}$, YUFENG LI ${ }^{1}$ and JINGHUA ZHANG ${ }^{1}$ \\ ${ }^{1}$ The Cancer Institute, Departments of ${ }^{2}$ Nuclear Medicine Clinical Laboratory and ${ }^{3}$ Neurosurgery, \\ Tangshan People's Hospital, Tangshan, Hebei 063001; ${ }^{4}$ College of Life Sciences, North China \\ University of Science and Technology, Tangshan, Hebei 063000, P.R. China
}

Received October 26, 2015; Accepted March 3, 2017

DOI: $10.3892 / \mathrm{ol} .2017 .6348$

\begin{abstract}
MicroRNAs (miRs) have been reported to be associated with the development of numerous types of cancer. However, the function of miRs in human ovarian carcinoma chemoresistance remains largely undefined. In the present study, cell chemotherapy combined with a Cell Counting Kit- 8 assay demonstrated that miR-20a performed important roles in ovarian cancer cells chemoresistance. Flow cytometry, cellular proliferation assays and Transwell assays results revealed that the proliferation and migration rates of OVCAR3/DDP cells were increased in comparison with parental cells. Western blot analysis results suggested that epithelial-mesenchymal transition (EMT) activated by miR-20a contributed to OVCAR3/DDP cell migration. The present study highlighted the importance of miR-20a in regulating the chemoresistant properties of OVCAR3 cells and promoting cisplatin-resistant cell migration by activating EMT. The results of present study may therefore provide novel insights into reversing the chemoresistance of ovarian cancer and improving its treatment.
\end{abstract}

\section{Introduction}

Ovarian cancer is the fifth most common type of disease in females, and in the United States in 2014 there were an estimated 21,290 new cases and 14,180 mortalities due to ovarian cancer (1). Although the 5-year survival rate of females with ovarian cancer has improved, it is only 20\% (2). Platinum-based combinations of chemoresistance is one of the obstacles limiting the success of cancer drug treatments and minimizing the effectiveness of chemotherapy in a large

Correspondence to: Professor Yufeng Li or Professor Jinghua Zhang, The Cancer Institute, Tangshan People's Hospital, 65 Sheng-Li Road, Tangshan, Hebei 063001, P.R. China

E-mail: fengfly01@163.com

E-mail: jinghuazhang2010@yahoo.com

Key words: ovarian cancer, microRNA-20a, cisplatin, resistance, migration number of patients (3). Cisplatin, one of the most common forms of platinum, is often used as one of the first-lines of treatment following surgical resection of visible nidus in ovarian cancer (3). In order to improve patient outcomes, it is critical to overcome cisplatin resistance of ovarian cancer cells (4).

Epigenetic changes at the molecular and cellular levels contributing to cisplatin-resistance have previously been reported, including alterations of platinum-DNA adducts, impairment in the apoptotic response of cells to adduct products, DNA methylation status transformation, histone modification and microRNAs (miRs) $(5,6)$. miRs are reported to be involved in the regulation of various biological processes, including embryonic development, cellular proliferation, differentiation, migration and apoptosis $(7,8)$. Studies have suggested that aberrant miR expression levels have been associated with tumor biology, including resistance to various chemotherapeutic agents $(9,10)$. For example, let-7b suppression induces resistance to cisplatin by the upregulation of cyclin D1 in glioblastoma (11). miRs overexpression has also been demonstrated to result in resistance to drugs in colorectal and prostate cancer $(12,13)$. miR-522 expression level was reduced in doxorubicin (DOX) resistant colon HT29 cell line and affected the sensitivity of the cells to DOX treatment by targeting ABCB5 (14). miR-200b has been shown to enhance chemosensitivity in prostate cancer via the regulation of Bmi-1 (15). miR-20a, a member of the miR-17-92 cluster, acts as a transforming growth factor $\beta$ receptor 2 suppressor for reverting cisplatin-resistance and inhibiting metastasis in non-small cell lung cancer (16). Of note, miR-20a also inhibited the pro-apoptotic activity and induced chemoresistance in leukemia cells (17). Our previous study demonstrated that miR-20a promoted proliferation and invasion by targeting the amyloid precursor protein in the ovarian cancer OVCAR3 cell line (18). The present study hypothesized that miR-20a may be involved in in ovarian cancer resistance to cisplatin and aimed to investigate the underlying mechanism of chemoresistance in OVCAR3 cells. A cisplatin-resistant subline, OVCAR3/DDP, was established from the OVCAR3 ovarian cell line. miR-20a facilitated OVCAR3 cells resistance to cisplatin and contributed to OVCAR3/DDP cell migration. The enhanced migration ability of OVCAR3/DDP cells may be due to epithelial-mesenchymal transition (EMT) induced by miR-20a. 


\section{Materials and methods}

Cell culture and transfection. Cells were routinely cultured in RPMI-1640 medium supplemented with $10 \%$ fetal bovine serum (FBS; Gibco; Thermo Fisher Scientific, Inc., Waltham, MA, USA), $100 \mathrm{IU} / \mathrm{ml}$ penicillin and $100 \mu \mathrm{g} / \mathrm{ml}$ streptomycin, and incubated at $37^{\circ} \mathrm{C}$ in a humidified chamber supplemented with $5 \% \quad \mathrm{CO}_{2}$ until confluence reached 70-80\%. Transfection was performed using Lipofectamine ${ }^{\mathrm{TM}} 2000$ reagent (Invitrogen; Thermo Fisher Scientific, Inc.), according to the manufacturer's protocol.

Plasmid construction. To construct the overexpression and control plasmids, the sequences of miR-20a precursor (sh-miR-20a) and control (NC-miR-20a) were subcloned into pcDNA3.1 polyclone sites with HindIII and BamHI sites, pri-miR-10a and pcDNA3, respectively. To knockdown the miR-20a expression, the sequence of miR-20a inhibitor (ASO-miR-20a) and control were synthesized (Gene Pharma, Shanghai, China). The sequences used are indicated in Table I.

Establishment of OVCAR3/DDP cell lines. OVCAR3/DDP cells were induced using a progressive concentration of cisplatin. Briefly, OVCAR3 that were sourced from Tianjin Medical University (Tianjin, China) cells in the logarithmic growth phase were treated with $2.5 \mu \mathrm{mol} / 1$ cisplatin. Following 48-72 h, cisplatin was removed and the cells were cultured at $37^{\circ} \mathrm{C}$ without cisplatin for $\sim 3$ weeks until they recovered. Then the cells were treated with $5 \mu \mathrm{mol} / 1$ and $10 \mu \mathrm{mol} / 1$ cisplatin, respectively. The cisplatin resistant OVCAR3/DDP cell line was successfully established when cells survived in $10 \mu \mathrm{mol} / 1$ cisplatin for $\sim 2$ months with a normal activity.

Cell Counting Kit-8 (CCK-8) assay. Cell viability was assessed using CCK-8 (Dojindo Molecular Technologies, Inc., Kumamoto, Japan). The absorbance at $450 \mathrm{~nm}$ was detected using a Thermo Scientific Mulyiskan FC Microplate Spectrophotometer. For the inhibition detection, the OVCAR3 and OVCAR3/DDP cells were treated with 5 different concentrations $(0,0.25,2.5,25,250,2,500 \mu \mathrm{mol} / \mathrm{l})$ for $48 \mathrm{~h}$, and then added with CCK-8. Inhibition $(\%)=\{1-$ [OVCAR3/DDP optical density (OD) - Blank OD]/(OVCAR3 OD - Blank OD) $\}$ x $100 \%$. Resistance index = OVCAR3/DDP half-maximal inhibitory concentration $\left(\mathrm{IC}_{50}\right) / \mathrm{OVCAR} 3 \mathrm{IC}_{50}$.

Cell cycle analysis. Cells were seeded in $25 \mathrm{~cm}^{2}$ tissue culture flasks at a density of $25 \times 10^{4} /$ well in the corresponding growth medium, and harvested when they reached $80 \%$ confluence. Subsequently, cells were washed with PBS twice and fixed in ice-cold $70 \%$ ethanol in PBS for $24 \mathrm{~h}$ at $4^{\circ} \mathrm{C}$. Subsequent to washing, the fixed cells were treated with $0.01 \%$ RNase for $10 \mathrm{~min}$ at $37^{\circ} \mathrm{C}$ and then stained with $0.05 \%$ propidium iodide (PI) for $20 \mathrm{~min}$ at $4^{\circ} \mathrm{C}$ in the dark. The cell cycle was determined using a FACScanto flow cytometer (BD Biosciences, Franklin Lakes, NJ, USA) and analyzed using ModFit software v.4.1 (Verity Software House, Inc., Topsham, ME, USA).

RNA isolation and reverse transcription-quantitative polymerase chain reaction $(R T-q P C R)$. The total RNA
Table I. Primer sequences for reverse transcription-quantitative polymerase chain reaction.

\begin{tabular}{lcl}
\hline Gene & Primer & \multicolumn{2}{c}{ Sequence } \\
\hline miR-20a & F & GCTGCCGTAAAGTGCTTATAGTG \\
& R & CAGAGCAGGGTCCGAGGTA \\
miR-20a & & CTACCTGCACTATAAGCACTTTA \\
inhibitor & & \\
miR-20a & & GACTACACAAATCAGCGATTT \\
control & & \\
sh-miR- & F & UAAAGUGCUUAUAGUGCAGGUAGTT \\
20a & R & CUACCUGCACUAUAAGCACUUUAT \\
sh-NC & F & UUCUCCGAACGUGUCACGUTT \\
U6 & R & ACG UGACACGUUCGGAGAATT \\
& F & ATTGGAACGATACAGAGAAGATT \\
& R & GGAACGCTTCACGAATTTG
\end{tabular}

F, forward; R, reverse; miR, microRNA; sh, short hairpin; NC, negative control.

of OVCAR3/DDP cells was isolated using EASYspin tissue/cell total RNA extraction kit (Aidlab Biotechnologies, Ltd., Beijing, China) and the cDNA was synthesized using the First-Strand cDNA Synthesis kit including DNase (Takara Bio., Inc., Otsu, Japan) from $3 \mu \mathrm{g}$ total RNA with a thermocycler (Arktik 96, Thermo Fisher Scientific, Inc.). miR expression level was analyzed by stem-loop RT-qPCR using Hairpin-itä miRs RT-PCR Quantitation kit (Shanghai GenePharma Co., Ltd., Shanghai, China) and SYBR Premix Ex Taq (Takara Bio, Inc.) on a PikoReal 96 Real-Time PCR System (Thermo Fisher Scientific, Inc.). The qPCR assay was performed as follows: $95^{\circ} \mathrm{C}$ for $3 \mathrm{~min}$ for 40 cycles of $95^{\circ} \mathrm{C}$ for $12 \mathrm{sec}$ and $62^{\circ} \mathrm{C}$ for $40 \mathrm{sec}$. The fold change was normalizing to U6 by using $2^{-\Delta \Delta \mathrm{Cq}}$ (19). The primer sequences are provided in Table I. All experiments were performed in triplicate.

Western blot analysis. Protein samples were obtained by lysing ovarian cancer OVCAR3/DDP cells in a standard sample buffer (50 mM Tris- $\mathrm{HCl}$; $\mathrm{pH}$ 6.8; $2 \% \mathrm{SDS} ; 10 \%$ glycerol) with level of shaking for $20 \mathrm{~min}$ at $4^{\circ} \mathrm{C}$, with gentle triturating 4 times. Lysates were collected and cleared by centrifugation at $12,830 \mathrm{x}$ g for $5 \mathrm{~min}$ at $4^{\circ} \mathrm{C}$. Then, $30 \mu \mathrm{g}$ protein of each group was loaded into $10 \%$ SDS-PAGE. The membranes were blocked with $5 \%$ bovine serum albumin (BSA; Amresco, LLC, Solon, OH, USA) at $37^{\circ} \mathrm{C}$ for $1 \mathrm{~h}$ and incubated with the specific antibodies diluted with $5 \%$ BSA at $4^{\circ} \mathrm{C}$ overnight. The membranes were washed with TBST 3 times for $5 \mathrm{~min}$, and then incubated with goat anti-rabbit peroxidase-conjugated secondary antibody for $2 \mathrm{~h}$ at $37^{\circ} \mathrm{C}$. The following primary antibodies were used at the indicated dilutions: Anti-E-cadherin pAb (dilution, 1:500; cat. no. sc-7870), anti-N-cadherian pAb (dilution, 1:500; cat. no. sc-393933; both from Santa Cruz Biotechnology Inc., Dallas, TX, USA), anti-Vimentin (dilution, 1:500; cat. no. bs-8533R) and $\beta$-tubulin pAb (dilution, 1:1,000; cat. no. bs-14263R, both from Bioss Biological Technology Co., Ltd., Beijing, China). 

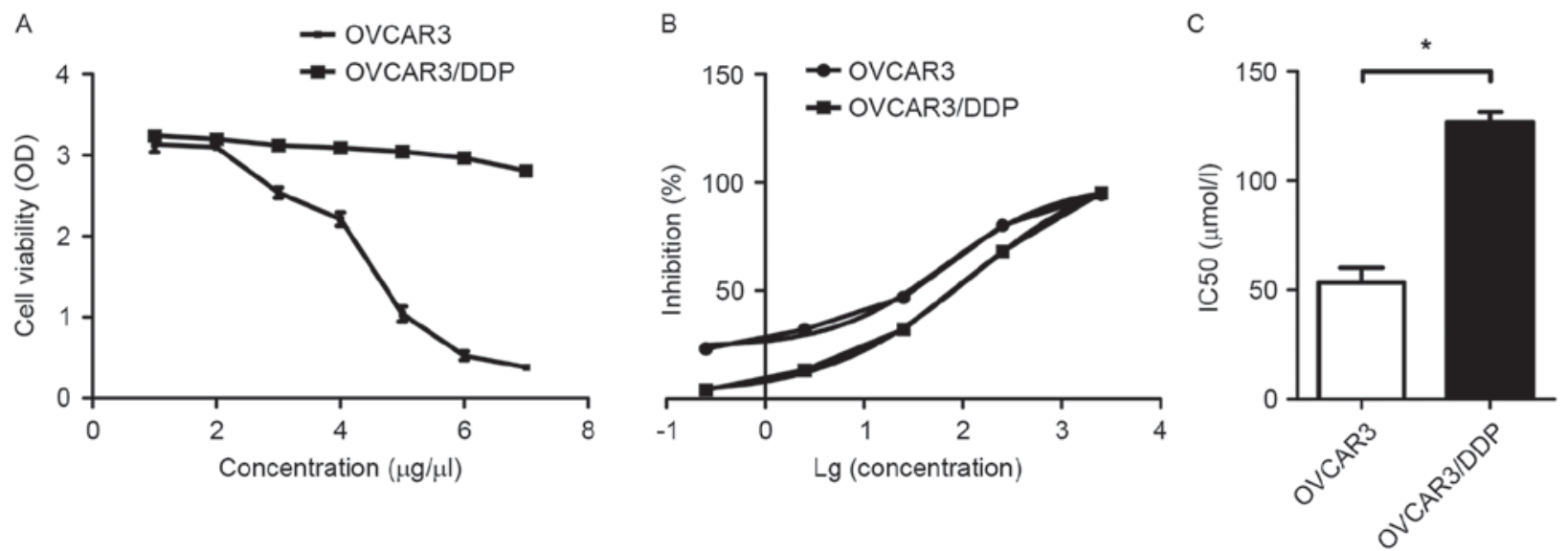

Figure 1. Establishment of the cisplatin-resistant cell line OVCAR3/DDP. (A) OVCAR3 and OVCAR3/DDP cell viability following treatment with various cisplatin concentrations. (B) The inhibition rate of cisplatin in OVCAR3 and OVCAR3/DDP cell lines. (C) The IC ${ }_{50}$ of OVCAR3 and OVCAR3/DDP cells was determined. ${ }^{*} \mathrm{P}<0.05$. $\mathrm{IC}_{50}$, half maximal inhibitory concentration; OD, optical density.

Boyden chamber transwell migration assay. The migratory capability of cells was determined using a Transwell chamber culture system ( $8 \mu \mathrm{m}$ pore; Corning Incorporated, Corning, NY, USA). Cells were seeded in a Boyden chamber Transwell without matrigel-coated inserts $\left(2 \times 10^{4} /\right.$ well $)$ with serum-free growth medium Opti-MEM (Gibco; Thermo Fisher Scientific, Inc.). Complete growth medium supplemented with $10 \% \mathrm{FBS}$ was added to the lower chamber. Following incubation at $37^{\circ} \mathrm{C}$ for $24 \mathrm{~h}$, the cells attached to the lower surface of the insert filter were counted following $0.1 \%$ crystal violet (Solarbio Science \& Technology Co., Ltd, Beijing, China) staining for 10 mins at room temperature.

Statistical analysis. All experiments were repeated independently $\geq 3$ times, and the results are presented as the mean \pm standard deviation. A Student t-test was used to evaluate the statistical differences between the two groups. $\mathrm{P}<0.05$ was considered to indicate a statistically significant difference.

\section{Results}

Establishment of cisplatin-resistant human ovarian cancer cell line OVCAR3/DDP. Cisplatin-resistant capability of OVCAR3/DDP cells and the parental cells was assessed using a CCK-8 assay at various concentrations of cisplatin. As presented in Fig. 1A, OVCAR3 cell viability was gradually decreased and almost completely inhibited following treatment with $7 \mu \mathrm{mol} / 1$ cisplatin. OVCAR3/DDP cell viability was slightly decreased following the same concentration treatment. Subsequently, the $\mathrm{IC}_{50}$ values for the two types of cells were detected using CCK- 8 assay with 5 concentrations of cisplatin $(0.25,2.5,25,250$ and $2,500 \mu \mathrm{mol} / \mathrm{l})$. The growth inhibition rate of OVCAR3/DDP cells was lower than that of OVCAR3 cells at each concentration, except 2,500 $\mu \mathrm{mol} / 1$, at which the two cell lines were almost entirely inhibited (Fig. 1B). The $\mathrm{IC}_{50}$ of OVCAR3/DDP and OVCAR3 cells were $122.30 \pm 2.83$ and $66.08 \pm 5.25$, respectively, and the index (RI) of OVCAR3/DDP cells was 1.87 (Fig. 1C). These results indicated that OVCAR3/DDP cells had increased resistance to cisplatin compared with OVCAR3 cells.
Increased expression level of miR-20a contributes to cisplatin-resistance of $O V C A R 3 / D D P$ cells. It was reported that miR-20a was involved in drug-metastasis in various types of cancer cells $(16,20)$. In order to investigate whether miR-20a performs a role in cisplatin-resistant OVCAR3 cells, mRNA expression levels of miR-20a in OVCAR3 and OVCAR3/DDP cells were evaluated using RT-qPCR. As presented in Fig. 2A, miR-20a was overexpressed in OVCAR3/DDP cells compared with in the parent cells. In addition, overexpression of miR-20a (pri-miR-20a) in OVCAR3 could enhance resistance to cisplatin compared with in the control group (pcDNA3; Fig. 2B). Knockdown of miR-20a by ASO-miR-20a could reduce resistant capability to cisplatin compared with the control in OVCAR3/DDP cells (Fig. 2C). These results demonstrated that miR-20a enhanced cisplatin resistance in OVCAR3/DDP cells.

The proliferation of OVCAR3/DDP cells is increased. In order to determine the proliferation of OVCAR3/DDP cells, a CCK-8 assay was used to detect the cell viability of OVCAR3 and OVCAR3/DDP cells for 7 consecutive days. The population doubling time was analyzed according to $\mathrm{T}_{\mathrm{d}}$ (doubling time $)=\Delta$ tx $\lg 2 /\left(\lg \mathrm{N}_{\mathrm{t}}-\lg \mathrm{N}_{0}\right)$; where $\mathrm{N}_{0}$ is the cell number at the beginning and $\mathrm{N}_{\mathrm{t}}$ is the cell number at the end, and $\Delta \mathrm{t}$ is the time from $\mathrm{N}_{0}$ to $\mathrm{N}_{\mathrm{t}}$. $\mathrm{T}_{\mathrm{d}}$ (OVCAR3) and $\mathrm{T}_{\mathrm{d}}$ (OVCAR3/DDP) were $\sim 88$ and $69 \mathrm{~h}$, respectively (Fig. 3A), which suggested that OVCAR3/DDP cells acquired a reinforced proliferation compared with OVCAR3 cells. In addition, flow cytometry assay results demonstrated that there were fewer OVCAR3/DDP cells in the $G_{0} / G_{1}$ cell cycle phase and an increased number of cells in the $S$ and $G_{2} M$ phases (Fig. 3B and C). The proliferation index of OVCAR3/DDP and OVCAR3 cells was 40.59 and $30.73 \%$, respectively, which suggested that miR-20a may promote OVCAR3/DDP cellular proliferation.

miR-20a regulates OVCAR3 and OVCAR3/DDP cell migration. The present study verified the migration abilities of OVCAR3 and OVCAR3/DDP cells. The migration ability of OVCAR3/DDP cell was enhanced $155 \%$ compared with OVCAR3 cells (Fig. 4A and B). To determine whether miR-20a had an effect on the migration of cells, a Transwell assay was performed to determine the migration of 

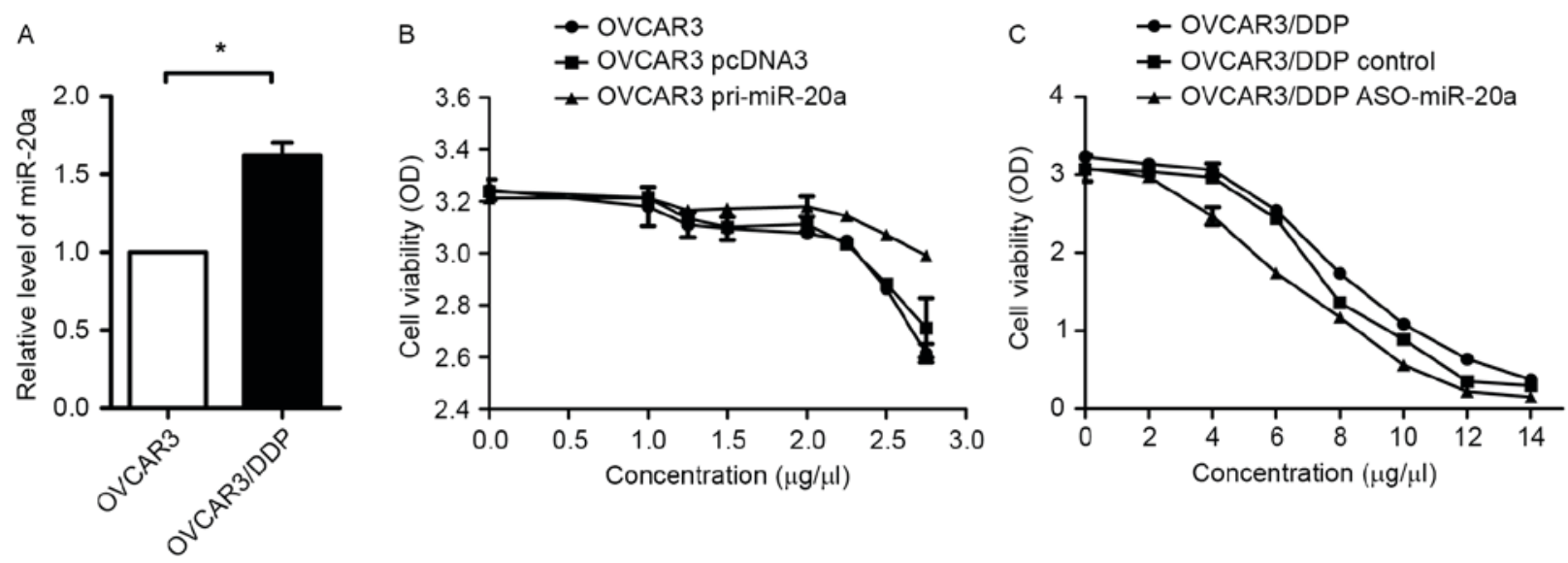

Figure 2. miR-20a contributes to cisplatin-resistance of OVCAR3/DDP cells. (A) miR-20a expression level of OVCAR3 and OVCAR3/DDP cells. (B) Cell viability of OVCAR3 cells transfected with pri-miR-20a (miR-20a overexpression) and pcDNA3 (control) at various concentrations of cisplatin. (C) Cell viability of OVCAR3/DDP transfected with ASO-miR-20a (miR-20a downregulation) and control at various concentrations of cisplatin. "P<0.05. OD, optical density; miR, microRNA.
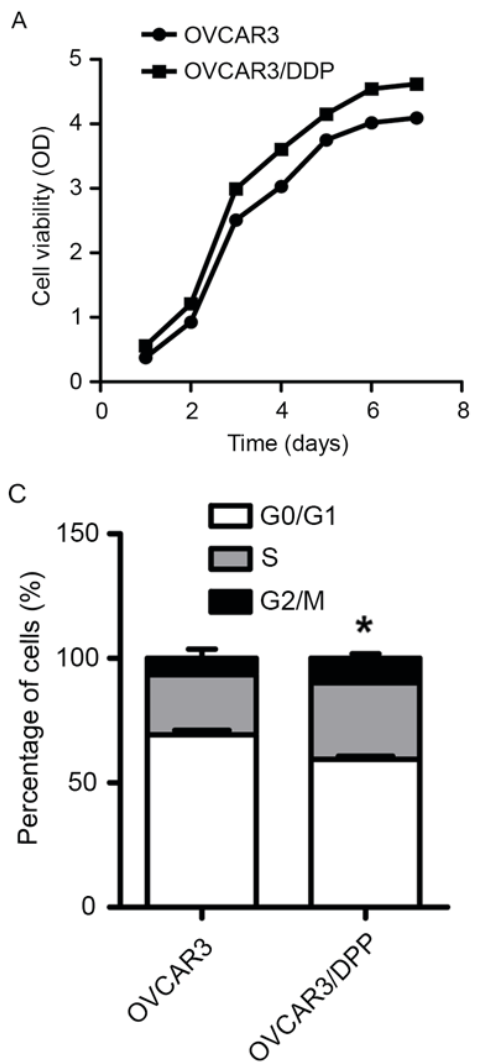

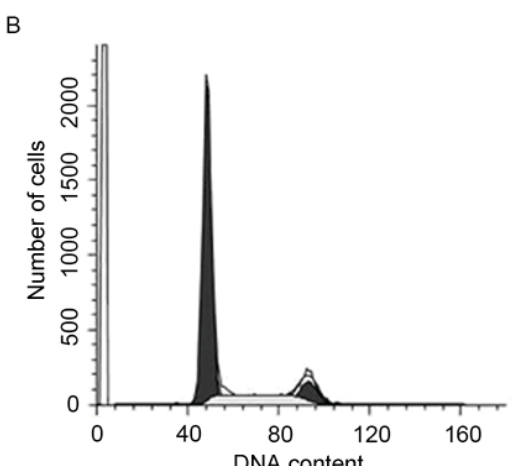

OVCAR3

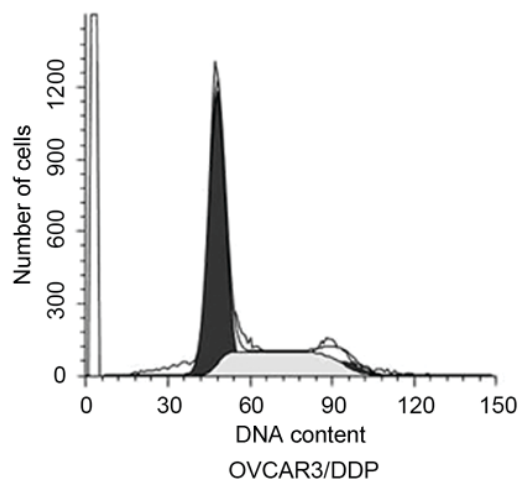

Figure 3. The proliferation ability of OVCAR3/DDP cells is enhanced. (A) Cell viability of OVCAR3 and OVCAR3/DDP cells was determined using the Cell Counting Kit-8 assay. (B) Cell cycle distribution of OVCAR3 and OVCAR3/DDP cells examined by flow cytometry assay. (C) Histograms represent flow cytometry data for cell populations in G0/G1, S and G2/M stages of the cell cycle. The data represents $\geq 3$ evaluations. " $\mathrm{P}<0.05$. OD, optical density.

OVCAR 3 cells transfected with pri-miR-20a or pcDNA3 and OVCAR3/DDP cells transfected with ASO-miR-20a or the control. An increased or decreased number of migrated cells were observed corresponding to upregulation or downregulation of miR-20a (Fig. 4C and D).

OVCAR3/DDP cells undergo EMT. During the cisplatin-resistant cells development, OVCAR3 cells exhibited protrusion and mesenchymal phenotypes (Fig. 5A). Thus, the present study evaluated the EMT markers of OVCAR3 and OVCAR3/DDP cells by western blot analysis. OVCAR3/DDP cells expressed increased levels of Vimentin and N-cadherin and decreased levels of E-cadherin compared with OVCAR3 cells (Fig. 5B), which suggested that EMT occurred during the process of cisplatin-resistance. In addition, OVCAR3/DDP cells transfected with ASO-miR-20a exhibited decreased expression levels of $\mathrm{N}$-cadherin and Vimentin protein and increased expression levels of E-cadherin protein, compared 
A

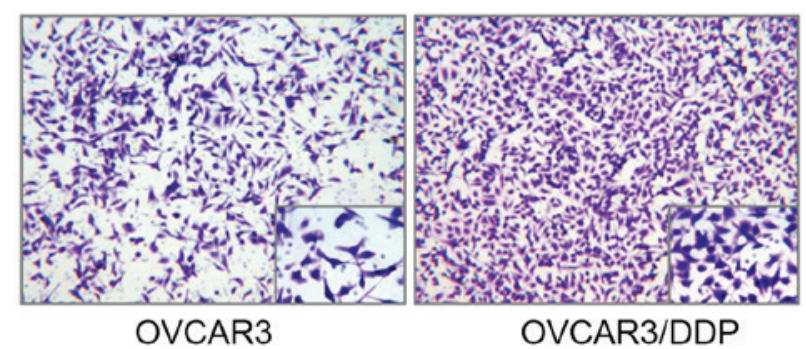

$\mathrm{C}$
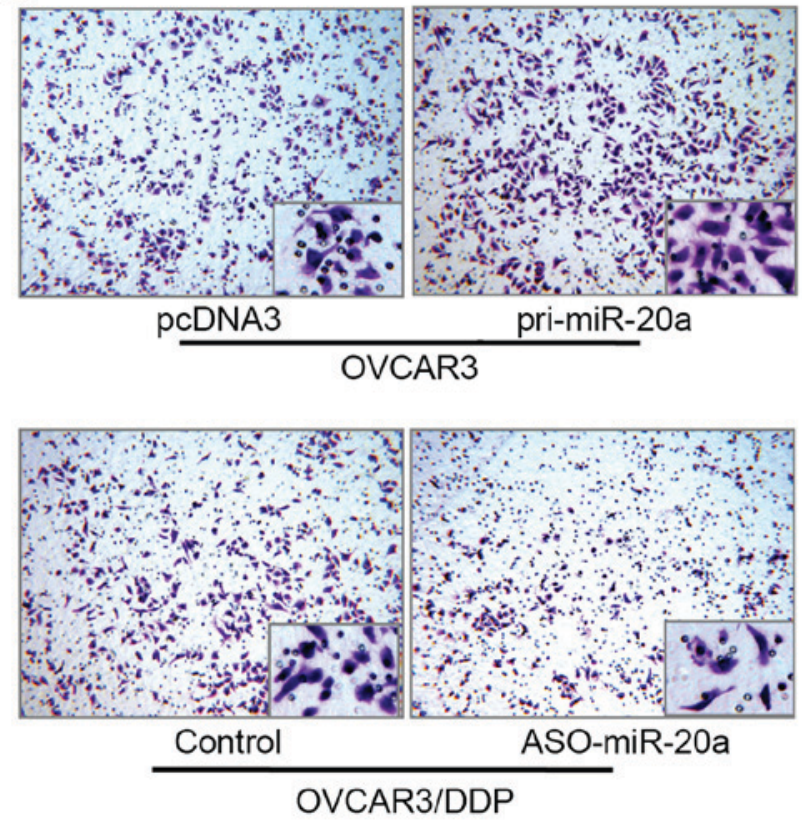

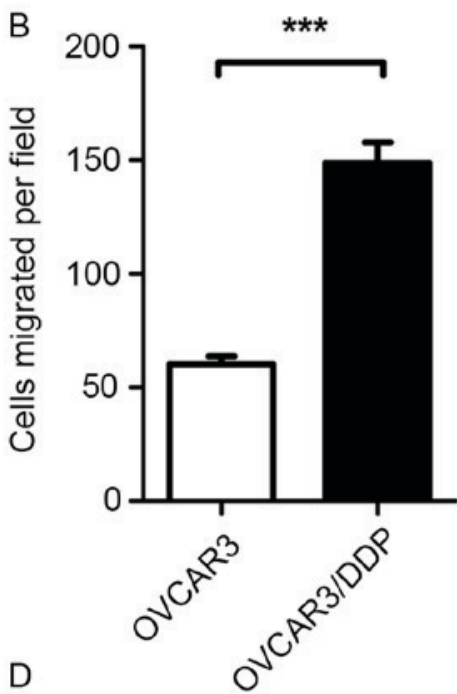

D
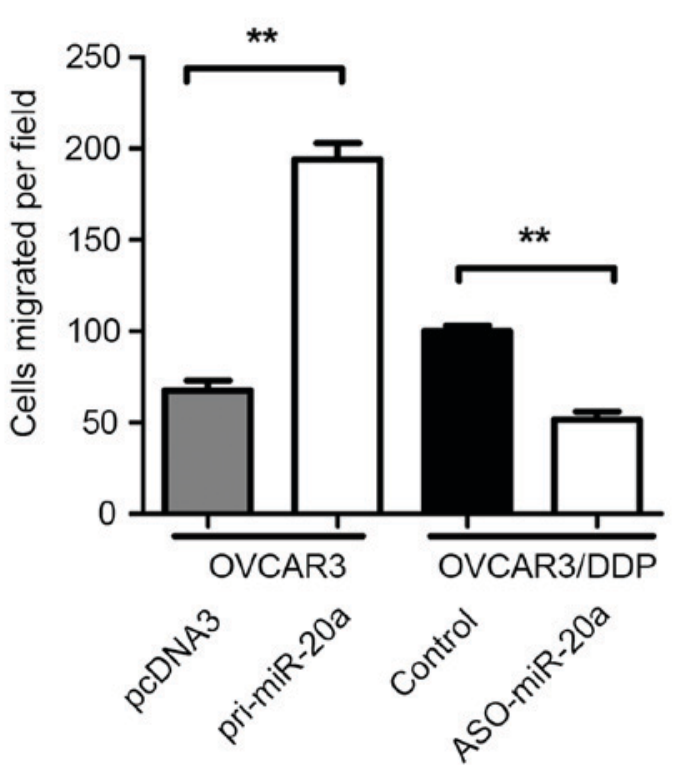

Figure 4. miR-20a regulates OVCAR3 and OVCAR3/DDP cells migration. (A) Migration activity of OVCAR3 and OVCAR3/DDP cells was detected by Transwell assay. The representative images are magnification, $x 100$. (B) Quantitative results of three independent experiments. The migrated cells were measured by software of Image-Pro Plus v.6.0 $\left({ }^{* * * *} \mathrm{P}<0.001\right)$. (C) Migration activity of OVCAR3 cells transfected with pri-miR-20a or pcDNA3 and OVCAR3/DDP cells transfected with ASO-miR-20a or the control was evaluated. Representative images of the photomicrographs were captured at magnification, x100. (D) The statistical results of the migrated cells are indicated $\left({ }^{* *} \mathrm{P}<0.01\right)$. miR, microRNA.

with the control group (Fig. 5C and D). These results suggested that miR-20a may induce EMT in OVCAR3/DDP cells.

\section{Discussion}

Ovarian cancer is the most lethal type of gynecological cancer (21). Although the international standard of care is surgery supplemented with paclitaxel and platinum based chemotherapy, the majority of patients will relapse in one to two years due to chemoresistance (22). Cisplatin is one of the most commonly used platinum based chemotherapy treatments for ovarian cancer; however, the resistant mechanism underlying cisplatin cytotoxicity to cancer cells remains unclear. Additional understanding of the mechanism underlying ovarian cancer resistance to cisplatin may aid the development of extending the survival rate and increasing the percentage of females who are cured from ovarian cancer. In the present study, a cisplatin-resistant subline, OVCAR3/DDP, was established from OVCAR3 ovarian cancer cells. The proliferation and migration abilities of OVCAR3/DDP were enhanced compared with those of OVCAR 3 cells.

Dysregulation of miRs has been widely documented in almost all types of human malignancies (23). Certain miRs may improve chemotherapy sensitivity, whereas others may induce chemoresistance of cancer cells (24-26). In ovarian cancer, miR-130a and miR-374a were demonstrated to function as negative regulators of cisplatin resistance in A2780 cells (27), whereas miR-31 positively regulated cisplatin resistance in numerous ovarian cancer cells (28). In the present study, miR-20a was identified as a novel positive regulator for OVCAR3 cells cisplatin resistance and migration. Previous studies have suggested that miRs may induce EMT development, drug resistance and metastasis (29-32). During EMT, epithelial 
A

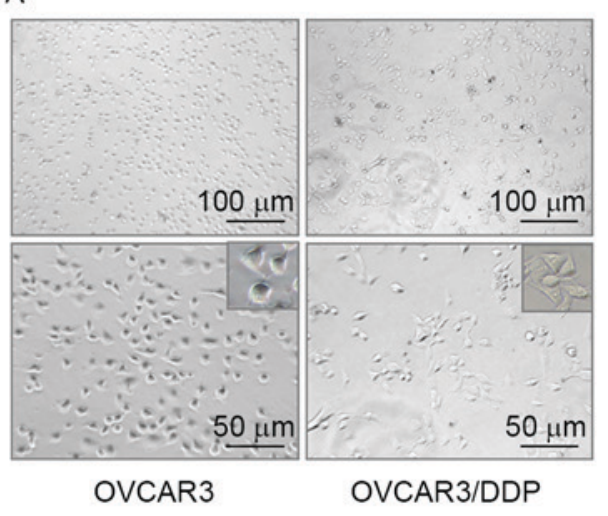

C

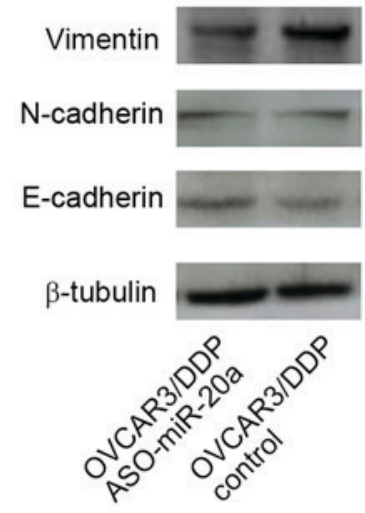

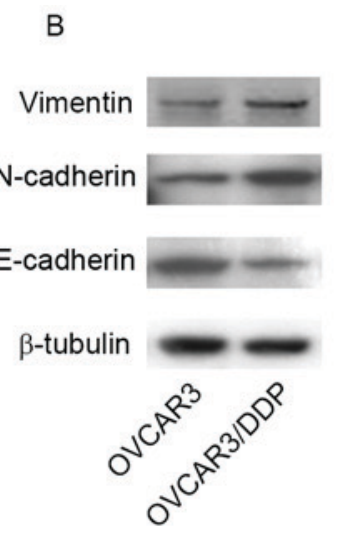

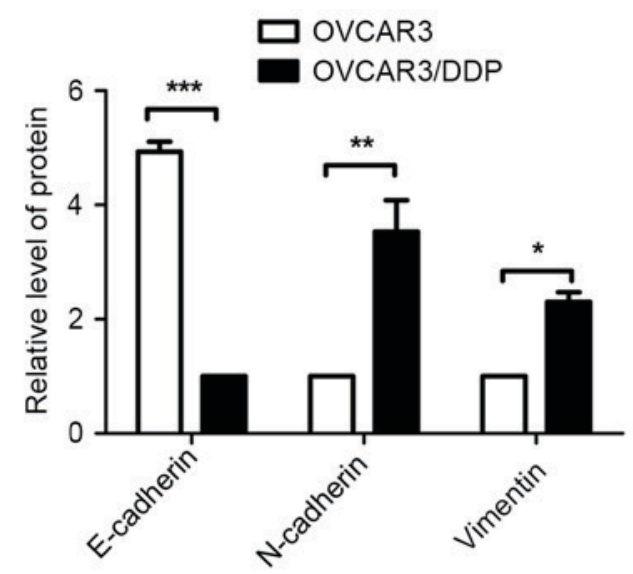

D

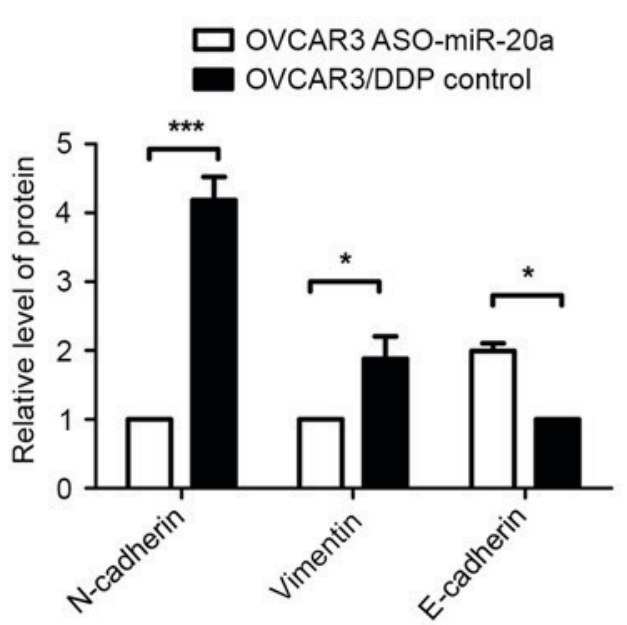

Figure 5. Epithelial-mesenchymal transition occurred in OVCAR3/DDP cells. (A) Morphology of OVCAR3 and OVCAR3/DDP cells. (B) The expression levels of Vimentin, E-cadherin and N-cadherin in OVCAR3 and OVCAR3/DDP cells were determined by western blotting and normalized to that of $\beta$-tubulin. Bars indicated the normalized mean intensity \pm standard deviation for three independent experiments. (C) The expression levels of Vimentin, E-cadherin and N-cadherin in OVCAR3/DDP cells transfected with ASO-miR-20a and the control. (D) The relative histogram of the expression levels of Vimentin, E-cadherin and N-cadherin in OVCAR3/DDP cells transfected with ASO-miR-20a and the control ( $\left.\mathrm{P}<0.05,{ }^{* * *} \mathrm{P}<0.01,{ }^{* * * *} \mathrm{P}<0.001\right)$. miR, microRNA.

cells acquire a mesenchymal phenotype that is characterized by the loss of intercellular junctions and increased cell migration (33). In the present study, OVCAR3/DDP cells morphology was altered due to cisplatin, and the cells expressed increased levels of E-cadherin and decreased levels of Vimentin and $\mathrm{N}$-cadherin, compared with the parental cells, which suggested that EMT occurred during the cells cisplatin-resistance. In addition, the effects of the EMT markers were reversed by inhibition of the expression of miR-20a. These results indicated that EMT in OVCAR3/DDP cells may be activated by miR-20a, which accelerated the ovarian cancer malignant development.

The present study provided a novel insight into understanding the mechanism underlying chemoresistance of ovarian cancer cells. It also suggested that miR-20a may be promising as a novel therapeutic target for overcoming drug resistance and metastasis of ovarian cancer.

\section{Acknowledgements}

The authors would like to thank of Department of Pathogen Biology of Tianjin Medical University for donating the OVCAR3 cell line. The present study was supported by the National Natural Science Foundation of China (grant no. 81301779).

\section{References}

1. Jayson GC, Kohn EC, Kitchener HC and Ledermann JA: Ovarian cancer. Lancet 384: 1376-1388, 2014.

2. Colombo N, Peiretti M, Parma G, Lapresa M, Mancari R, Carinelli S, Sessa C and Castiglione M; ESMO Guidelines Working Group: Newly diagnosed and relapsed epithelial ovarian carcinoma: ESMO clinical practice guidelines for diagnosis, treatment and follow-up. Ann Oncol 21 (Suppl 5): v23-v30, 2010.

3. Foster T, Brown TM, Chang J, Menssen HD, Blieden MB and Herzog TJ: A review of the current evidence for maintenance therapy in ovarian cancer. Gynecol Oncol 115: 290-301, 2009.

4. Morgan RJ Jr, Alvarez RD, Armstrong DK, Burger RA, Chen LM, Copeland L, Crispens MA, Gershenson DM, Gray HJ, Hakam A, et al: Ovarian cancer, version 2.2013. J Natl Compr Canc Netw 11: 1199-1209, 2013.

5. Shen DW, Pouliot LM, Hall MD and Gottesman MM: Cisplatin resistance: A cellular self-defense mechanism resulting from multiple epigenetic and genetic changes. Pharmacol Rev 64: 706-721, 2012.

6. Borley J and Brown R: Epigenetic mechanisms and therapeutic targets of chemotherapy resistance in epithelial ovarian cancer. Ann Med 47: 359-369, 2015.

7. Bartel DP: MicroRNAs: Genomics, biogenesis, mechanism, and function. Cell 116: 281-297, 2004.

8. Anglicheau D, Muthukumar T and Suthanthiran M: MicroRNAs: Small RNAs with big effects. Transplantation 90: 105-112, 2010.

9. Xu C, Xie S, Song C, Huang L and Jiang Z: Lin28 mediates cancer chemotherapy resistance via regulation of miRNA signaling. Hepatogastroenterology 61: 1138-1141, 2014. 
10. Niu J, Xue A, Chi Y, Xue J, Wang W, Zhao Z, Fan M, Yang CH, Shao ZM, Pfeffer LM, et al: Induction of miRNA-181a by genotoxic treatments promotes chemotherapeutic resistance and metastasis in breast cancer. Oncogene 35: 1302-1313, 2016.

11. Guo Y, Yan K, Fang J, Qu Q, Zhou M and Chen F: Let-7b expression determines response to chemotherapy through the regulation of cyclin D1 in glioblastoma. J Exp Clin Cancer Res 32: 41, 2013.

12. Xie T, Huang M, Wang Y, Wang L, Chen $\mathrm{C}$ and Chu $\mathrm{X}$ : MicroRNAs as regulators, biomarkers and therapeutic targets in the drug resistance of colorectal cancer. Cell Physiol Biochem 40: 62-76, 2016.

13. Kopczynska E: Role of microRNAs in the resistance of prostate cancer to docetaxel and paclitaxel. Contemp Oncol (Pozn) 19: 423-427, 2015

14. Yang G, Jiang O, Ling D, Jiang X, Yuan P, Zeng G, Zhu J, Tian J, Weng $\mathrm{Y}$ and Wu D: MicroRNA-522 reverses drug resistance of doxorubicin-induced HT29 colon cancer cell by targeting ABCB5. Mol Med Rep 12: 3930-3936, 2015.

15. Yu J, Lu Y, Cui D, Li E, Zhu Y, Zhao Y, Zhao F and Xia S: miR-200b suppresses cell proliferation, migration and enhances chemosensitivity in prostate cancer by regulating Bmi-1. Oncol Rep 31: 910-918, 2014.

16. Jiang Z, Yin J, Fu W, Mo Y, Pan Y, Dai L, Huang H, Li S and Zhao J: MiRNA 17 family regulates cisplatin-resistant and metastasis by targeting TGFbetaR2 in NSCLC. PloS One 9: e94639, 2014.

17. Weng H, Huang H, Dong B, Zhao P, Zhou H and Qu L: Inhibition of miR-17 and miR-20a by oridonin triggers apoptosis and reverses chemoresistance by derepressing BIM-S. Cancer Res 74: 4409-4419, 2014.

18. Fan X, Liu Y, Jiang J, Ma Z, Wu H, Liu T, Liu M, Li X and Tang H: miR-20a promotes proliferation and invasion by targeting APP in human ovarian cancer cells. Acta Biochim Biophys Sin (Shanghai) 42: 318-324, 2010.

19. Livak KJ and Schmittgen TD: Analysis of relative gene expression data using real-time quantitative PCR and the 2(-Delta Delta C(T)) method. Methods 25: 402-408, 2001.

20. Zhang Y, Zheng L, Ding Y, Li Q, Wang R, Liu T, Sun Q, Yang H, Peng S, Wang W and Chen L: MiR-20a induces cell radioresistance by activating the PTEN/PI3K/Akt signaling pathway in hepatocellular carcinoma. Int J Radiat Oncol Biol Phys 92: 1132-1140, 2015

21. Jayson GC, Kohn EC, Kitchener HC and Ledermann JA: Ovarian cancer. Lancet 384: 1376-1388, 2014.
22. Markman M: Current standards of care for chemotherapy of optimally cytoreduced advanced epithelial ovarian cancer. Gynecol Oncol 131: 241-245, 2013.

23. Calin GA, Sevignani C, Dumitru CD, Hyslop T, Noch E, Yendamuri S, Shimizu M, Rattan S, Bullrich F, Negrini M and Croce CM: Human microRNA genes are frequently located at fragile sites and genomic regions involved in cancers. Proc Natl Acad Sci USA 101: 2999-3004, 2004

24. Garzon R, Calin GA and Croce CM: MicroRNAs in cancer. Annu Rev Med 60: 167-179, 2009.

25. Iorio MV and Croce CM: MicroRNAs in cancer: Small molecules with a huge impact. J Clin Oncol 27: 5848-5856, 2009.

26. Farazi TA, Spitzer JI, Morozov P and Tuschl T: miRNAs in human cancer. J Pathol 223: 102-115, 2011.

27. Li N, Yang L, Wang H, Yi T, Jia X, Chen C and Xu P: MiR-130a and MiR-374a function as novel regulators of cisplatin resistance in human ovarian cancer A2780 cells. PloS One 10: e0128886, 2015.

28. Samuel P, Pink RC, Caley DP, Currie JM, Brooks SA and Carter DR: Over-expression of miR-31 or loss of KCNMA1 leads to increased cisplatin resistance in ovarian cancer cells. Tumour Biol 37: 2565-2573, 2016.

29. Song J and Li Y: miR-25-3p reverses epithelial-mesenchymal transition via targeting Sema4C in cisplatin-resistance cervical cancer cells. Cancer Sci 108: 23-31, 2017.

30. Raza U, Saatci Ö, Uhlmann S, Ansari SA, Eyüpoğlu E, Yurdusev E, Mutlu M, Ersan PG, Altundağ MK, Zhang JD, et al: The miR-644a/CTBP1/p53 axis suppresses drug resistance by simultaneous inhibition of cell survival and epithelial-mesenchymal transition in breast cancer. Oncotarget 7: 49859-49877, 2016.

31. Singh R, Yadav V, Kumar S and Saini N: MicroRNA-195 inhibits proliferation, invasion and metastasis in breast cancer cells by targeting FASN, HMGCR, ACACA and CYP27B1. Sci Rep 5: $17454,2015$.

32. Zuo QF, Cao LY, Yu T, Gong L, Wang LN, Zhao YL, Xiao B and Zou QM: MicroRNA-22 inhibits tumor growth and metastasis in gastric cancer by directly targeting MMP14 and Snail. Cell Death Dis 6: e2000, 2015.

33. Kraljevic Pavelic S, Sedic M, Bosnjak H, Spaventi S and Pavelic K: Metastasis: New perspectives on an old problem. Mol Cancer 10: 22, 2011. 